\title{
Epirrubicina no tratamento do câncer de mama
}

\author{
Epirubicin in breast cancer treatment
}

\author{
Everardo D. Saad,' Gil Facina² e Luiz Henrique Gebrim ${ }^{2}$
}

\section{Resumo}

A epirrubicina, um análogo estrutural da doxorrubicina, pode ser empregada nas várias fases do tratamento quimioterápico do câncer de mama. A epirrubicina possui a vantagem de causar menor toxicidade cardíaca do que a doxorrubicina, quando são comparadas doses habitualmente consideradas como semelhantes do ponto de vista de eficácia. Apesar disso, ainda não está completamente estabelecida a equivalência de dose entre as duas antraciclinas. Os estudos em doenças localizada ou metastática sugerem que ambas podem ser empregadas de maneira intercambiável. Nos tratamentos curativo, em especial a quimioterapia adjuvante, ainda não foi definida a melhor dose ou o melhor esquema contendo a epirrubicina. Neste artigo, faz-se uma revisão do papel da epirrubicina no tratamento curativo e paliativo do câncer de mama, com ênfase maior na questão da dose deste quimioterápico que deve ser empregada no tratamento adjuvante. Discutem-se os estudos randomizados e as metanálises disponíveis até o presente momento e sugere-se o regime quimioterápico, contendo a epirrubicina, que mais parece adequado ao tratamento.

Palavras-chave: Antibióticos antineoplásicos; Antraciclinas; Doxorrubicina; Neoplasias mamárias; Quimioterapia.

${ }^{1}$ Grupo Multidisciplinar de Oncologia, Escola Paulista de Medicina/UNIFESP.

${ }^{2}$ Disciplina de Mastologia, Escola Paulista de Medicina/UNIFESP.

Endereço para correspondência: Everardo D. Saad. Grupo Multidisciplinar de Oncologia, Escola Paulista de Medicina/UNIFESP - Rua Pedro de Toledo, 715 - 04039-032 - São Paulo - Brasil. E-mail: esaad.gmo@unifesp.epm.br 


\section{INTRODUÇÃO}

O câncer de mama aflige aproximadamente 1 milhão de mulheres por ano no mundo ${ }^{1}$. No Brasil, foram estimados cerca de 49 mil casos para o ano 2005, o que torna o câncer de mama a neoplasia não-cutânea mais comum em nosso país ${ }^{2}$. Dados do Instituto Nacional de Câncer sugerem que, no Brasil, aproximadamente, metade das pacientes é diagnosticada com câncer de mama localizado (estádios I e II) ${ }^{3}$. Grande parte dessas pacientes tem indicação de tratamento sistêmico pósoperatório, que reduz os riscos de recidiva e mortalidade pelo câncer de mama ${ }^{4,5}$. Além disso, cerca de um terço das pacientes brasileiras tem doença localmente avançada (estádio III) ao diagnóstico ${ }^{3}$. Essas pacientes são comumente tratadas com regimes quimioterápicos préoperatórios (neo-adjuvantes) ou pós-operatórios (adjuvantes). Em muitos casos, os regimes quimioterápicos empregados no tratamento adjuvante ou neo-adjuvante incluem uma antraciclina (doxorrubicina ou epirrubicina). Neste artigo, faz-se uma revisão do papel da epirrubicina no tratamento adjuvante, neo-adjuvante e paliativo, com ênfase especial na questão da dose deste quimioterápico que deve ser empregada no tratamento curativo.

\section{EFICÁCIA PRÉ-CLÍNICA E TOXICIDADE}

A epirrubicina (4-epidoxorrubicina) é um análogo estrutural da doxorrubicina desenvolvido na Itália nos anos $80^{6}$. Estudos pré-clínicos sugeriram que a epirrubicina apresentava efeito citotóxico semelhante ao da doxorrubicina em diversas linhagens celulares ${ }^{7,8}$. Em estudos clínicos, a epirrubicina se mostrou ativa em vários tipos de tumores, com toxicidade aparentemente menor que aquela causada pela doxorrubicina ${ }^{9-13}$.

Em especial, a toxicidade cardíaca, que pode ser causada pela doxorrubicina, pareceu menor com a epirrubicina ${ }^{9,10,13}$. Num estudo com doses de epirrubicina variando entre $50 \mathrm{mg} / \mathrm{m}^{2}$ e $90 \mathrm{mg} / \mathrm{m}^{2}$ a cada 3 semanas, avaliaçôes ecocardiográficas seriadas sugeriram menor efeito cardiotóxico do que com a doxorrubicina ${ }^{14}$. Em comparação direta entre a doxorrubicina e a epirrubicina, demonstrou-se que a epirrubicina pode ser empregada em doses cumulativas até duas vezes superiores àquelas responsáveis pela toxicidade cardíaca pela doxorrubicina ${ }^{15,16}$. Estudos com biopsias de endomiocárdio confirmaram que a epirrubicina causa menos danos cardíacos que a doxorrubicina, quando são comparadas doses cumulativas semelhantes ${ }^{17}$. Apesar desses estudos, ainda é incerto se a epirrubicina é, de fato, menos cardiotóxica do que a doxorrubicina em doses antitumorais eqüipotentes.

\section{DOENÇA METASTÁTICA: EPIRRUBICINA OU DOXORRUBICINA?}

Ainda não foi elucidada a equivalência entre as doses de doxorrubicina e epirrubicina necessárias para produzir o mesmo efeito clínico. Aparentemente, a dose máxima tolerada de epirrubicina é o dobro da dose da doxorrubicina ${ }^{16}$. Em estudos clínicos com pacientes portadoras de câncer de mama metastático, a epirrubicina foi empregada em doses variáveis, sendo os regimes mais comuns aqueles com doses entre $50 \mathrm{mg} / \mathrm{m}^{2}$ e $100 \mathrm{mg} / \mathrm{m}^{2}$ a cada 3 semanas. Nesses regimes, a epirrubicina é freqüentemente combinada a outros quimioterápicos. Assim como ocorre com a doxorrubicina, existe uma clara relação entre a dose de epirrubicina e a resposta tumoral na doença metastática ${ }^{18}$. Por outro lado, os estudos não demonstraram diferenças claras entre a sobrevida das pacientes com doença metastática que receberam doses mais baixas ou mais altas de epirrubicina ${ }^{19-23}$, e parece haver um nível a partir do qual o incremento progressivo da dose não mais é acompanhado por aumento da eficácia ${ }^{24}$. Curiosamente, um estudo escandinavo que avaliou doses totais semelhantes de epirrubicina, mas administradas apenas uma vez por mês ou semanalmente, concluiu que o regime mensal é mais eficaz, tanto em respostas objetivas quanto em sobrevida global ${ }^{25}$.

Uma metanálise que envolveu 10 estudos randomizados, comparando a doxorrubicina e a epirrubicina no câncer de mama metastático, demonstrou que as taxas de resposta são ligeiramente inferiores para a epirrubicina, embora não tenha sido encontrada diferença estatisticamente significativa ( $44 \%$ versus $47 \%)^{26}$. Em seis estudos em que foi possível a avaliação de sobrevida, demonstrou-se que a doxorrubicina está associada a um aumento relativo da sobrevida de $13 \%$, com significância estatística limítrofe (hazard ratio de 1,13, com intervalo de confiança de $95 \%$ de 1,00 a 1,27). A metanálise confirmou a menor toxicidade da epirrubicina, em comparação com a doxorrubicina.

Com base no exposto, poder-se-ia concluir que, nas doses empregadas habitualmente, a doxorrubicina é ligeiramente mais eficaz do que a epirrubicina na doença metastática. Entretanto as doses de epirrubicina empregadas nos estudos incluídos na metanálise foram as mesmas que as doses de doxorrubicina em cinco dos 10 estudos. Nos outros estudos, a dose de epirrubicina foi apenas $25 \%$ a $50 \%$ maior do que a dose da 
doxorrubicina. Portanto, a metanálise não esclarece a questão da eficácia comparativa entre estas duas antraciclinas, já que a dose de epirrubicina empregada em parte dos estudos pode ter sido insuficiente. Além disso, a natureza paliativa do tratamento dessas pacientes deve ser considerada. Nessa situação, uma droga menos tóxica e com eficácia apenas ligeiramente inferior pode ser útil na prática clínica. Ademais, atualmente, estão disponíveis diversas outras drogas eficazes no tratamento de segunda ou terceira linha da doença avançada. Tais drogas foram introduzidas mais recentemente e não foram administradas à maioria das pacientes incluídas nos estudos mais antigos. Do ponto de vista prático, portanto, pode-se considerar a epirrubicina como uma opção segura e com eficácia semelhante à da doxorrubicina para o tratamento paliativo do câncer de mama.

\section{EPIRRUBICINA NO TRATAMENTO NEO-ADJUVANTE}

No âmbito específico do câncer de mama localmente avançado, um estudo pela European Organization for Research and Treatment of Cancer avaliou dois regimens contendo doses diferentes de epirrubicina ${ }^{27}$. Nesse estudo, 448 pacientes com doença localmente avançada (T4, N2/3 ou carcinoma inflamatório) foram randomizadas para seis ciclos de tratamento com o regime CEF (ciclofosfamida oral, $75 \mathrm{mg} / \mathrm{m}^{2}$ do dia 1 ao 14; epirrubicina, $60 \mathrm{mg} / \mathrm{m}^{2}$ nos dias 1 e 8 ; e fluorouracil, $500 \mathrm{mg} / \mathrm{m}^{2}$ nos dias 1 e 8 , a cada 4 semanas) ou o regime EC (epirrubicina, $120 \mathrm{mg} / \mathrm{m} 2$ no dia 1 ; e ciclofosfamida, $830 \mathrm{mg} / \mathrm{m}^{2}$ no dia 1 , a cada 2 semanas), este último com suporte hematopoético pela filgrastima (G-CSF). $\mathrm{O}$ estudo não revelou diferenças significativas entre as taxas de sobrevida livre de doença ou de sobrevida global nos dois grupos.

Outros dois estudos de tratamento neo-adjuvante avaliaram o papel da epirrubicina, em comparação com a doxorrubicina ${ }^{28}$ ou com o regime $\mathrm{CMF}^{29}$. No primeiro estudo, denominado TOPIC, 426 pacientes com tumores $\geq 3 \mathrm{~cm}$ receberam seis ciclos do regime AC (doxorrubicina, $60 \mathrm{mg} / \mathrm{m}^{2}$ e ciclofosfamida, $600 \mathrm{mg} / \mathrm{m}^{2}$, ambos no dia 1 a cada 3 semanas) ou do regime EcisF (epirrubicina, $60 \mathrm{mg} / \mathrm{m}^{2}$ e cisplatina, $60 \mathrm{mg} / \mathrm{m}^{2}$, ambas no dia 1 a cada 3 semanas; e fluorouracil, $200 \mathrm{mg} / \mathrm{m}^{2} /$ dia por 18 semanas em infusão contínua). Nesse estudo, as pacientes também receberam tamoxifeno durante a quimioterapia. O estudo TOPIC não revelou diferenças significativas entre as taxas de resposta, sobrevida livre de doença ou sobrevida global nos dois grupos ${ }^{28}$.

Num estudo conduzido na Itália, 205 pacientes com tumores $>2,5 \mathrm{~cm}$ ou linfonodos axilares comprometidos receberam quatro ciclos do regime CMF injetável (ciclofosfamida, $600 \mathrm{mg} / \mathrm{m}^{2}$, methotrexato, $40 \mathrm{mg} / \mathrm{m}^{2} \mathrm{e}$ fluorouracil, $600 \mathrm{mg} / \mathrm{m}^{2}$ ); ou quatro ciclos de tratamento rotativo (CMFEV), incluindo as mesmas drogas do regime CMF: a epirrubicina $\left(40 \mathrm{mg} / \mathrm{m}^{2}\right.$ nos dias 1 e 8$)$ e a vincristina. A seguir, as pacientes foram operadas e submetidas a mais três ciclos de tratamento adjuvante com CMF. Nesse estudo, a taxa de resposta clínica completa foi maior no grupo tratado com o regime rotativo ( $21 \%$ versus $12 \%, \mathrm{p}=0,08)$, mas atingiu significância estatística apenas no subgrupo de pacientes pré-menopausa ( $26 \%$ versus $4 \%, \mathrm{p}=0,004)$. Não foram relatados os resultados de sobrevida livre de doença ou sobrevida global ${ }^{29}$.

Os estudos disponíveis não esclarecem se a epirrubicina pode substituir a doxorrubicina no tratamento neo-adjuvante do câncer de mama, nem respondem à questão relativa quanto à melhor dose da epirrubicina a ser empregada. $\mathrm{Na}$ prática clínica, a escolha dos regimes será influenciada por diversos fatores, incluindo o estadiamento, as características da paciente e do tumor e a preferência pessoal do médico por regimes empregados no tratamento adjuvante, em que a epirrubicina foi mais bem avaliada.

\section{DOENÇA LOCALIZADA: EPIRRUBIIINA OU DOXORRUBIIINA?}

Não se tem conhecimento de estudos que tenham comparado diretamente a epirrubicina e a doxorrubicina no tratamento do câncer de mama precoce. No tratamento adjuvante de mulheres com câncer de mama localizado, a quimioterapia com antraciclinas promove discreto, porém estatisticamente significativo, aumento da sobrevida livre de doença e da sobrevida global, em comparação com regimes sem antraciclinas ${ }^{5,30}$. Embora a metanálise dos estudos randomizados não tenha fornecido uma comparação formal entre os regimes com doxorrubicina ou epirrubicina, seus resultados não sugerem que tal diferença seja de magnitude apreciável. De acordo com a atualização mais recente da metanálise, a epirrubicina foi empregada em $40 \%$ dos estudos de tratamento adjuvante e a doxorrubicina, em $60 \%{ }^{30}$. Assim, tanto a doxorrubicina quanto a epirrubicina fazem parte de regimes quimioterápicos atualmente usados no tratamento adjuvante de pacientes com câncer de mama precoce.

\section{EPIRRUBiCINA NO TRATAMENTO ADJUVANTE: EM QUE DOSE?}

A epirrubicina é comumente combinada à ciclofosfamida e ao fluorouracil no regime conhecido como FEC. Diversas variaçôes desse regime, mais 
utilizado na Europa e no Canadá, foram empregadas no tratamento adjuvante do câncer de mama. Algumas delas foram comparadas ao regime CMF, um dos esquemas quimioterápicos empregados no tratamento adjuvante do câncer de mama precoce ${ }^{31}$. Num estudo canadense, a combinação conhecida como CEF, em que a ciclofosfamida é empregada por via oral, foi superior ao regime CMF oral em pacientes pré-menopausa com linfonodos positivos ${ }^{32}$. No regime CEF, a epirrubicina foi administrada na dose de $60 \mathrm{mg} / \mathrm{m}^{2}$ nos dias 1 e 8 a cada 4 semanas.

Numa comparação direta entre regimes com doses distintas de epirrubicina, pesquisadores franceses trataram pacientes com 4 ou mais linfonodos positivos e pacientes com 1 a 3 linfonodos positivos (neste último caso em tumores com grau 2 ou 3 de Bloom e Richardson e que não expressavam receptores hormonais $)^{33}$. Nesse estudo, 565 pacientes foram randomizadas para receber seis ciclos de FEC 50 (fluorouracil, $500 \mathrm{mg} / \mathrm{m}^{2}$, epirrubicina, $50 \mathrm{mg} / \mathrm{m}^{2} \mathrm{e}$ ciclofosfamida, $500 \mathrm{mg} / \mathrm{m}^{2}$, todos no dia 1 a cada 3 semanas) ou FEC 100 (mesmas doses de ciclofosfamida e fluorouracil, com epirrubicina na dose de $100 \mathrm{mg} / \mathrm{m}^{2}$ ). O regime FEC 100 promoveu melhor sobrevida global e discreto aumento da toxicidade, em comparação com o regime FEC 50 (Tabela 1).

Em outro estudo, comparando diretamente regimes com diferentes doses de epirrubicina, pesquisadores da Bélgica trataram pacientes com linfonodos positivos e idade igual ou inferior a 70 anos $^{34}$. Nesse estudo, 787 pacientes foram randomizadas para receber um de três tratamentos adjuvantes: seis ciclos de CMF oral, oito ciclos de EC (epirrubicina, $60 \mathrm{mg} / \mathrm{m}^{2}$ e ciclofosfamida, $500 \mathrm{mg} / \mathrm{m}^{2}$, ambos no dia 1 a cada 3 semanas) ou oito ciclos de HEC (epirrubicina, $100 \mathrm{mg} / \mathrm{m}^{2}$ e ciclofosfamida, $830 \mathrm{mg} / \mathrm{m}^{2}$, ambos no dia 1 a cada 3 semanas). Não houve diferenças entre os regimes CMF e HEC, mas este último foi mais eficaz do que o regime EC (Tabela 2). Não houve diferenças significativas entre os perfis de toxicidade com os dois regimes com epirrubicina.

Tabela 2. Comparação direta entre os regimes HEC e EC ${ }^{34}$

\begin{tabular}{l|c|c|c|c}
\hline \multicolumn{1}{|c|}{ Parâmetro } & HEC & EC & $\mathrm{p}$ & $\begin{array}{c}\text { Hazard } \\
\text { Ratio* (IC 95\%) }\end{array}$ \\
\hline $\begin{array}{l}\text { Sobrevida } \\
\text { livre de } \\
\text { eventos aos } \\
3 \text { anos }\end{array}$ & $80 \%$ & $72 \%$ & 0,04 & $\begin{array}{c}0,73 \\
(0,54-0,99)\end{array}$ \\
\hline $\begin{array}{l}\text { Sobrevida } \\
\text { global aos } \\
3 \text { anos }\end{array}$ & $92 \%$ & $89 \%$ & 0,05 & $\begin{array}{c}0,69 \\
(0,47-1,00)\end{array}$ \\
\hline
\end{tabular}

$\left(^{*}\right)$ Hazard ratio para mortalidade entre HEC e EC; EC=epirrubicina $(60 \mathrm{mg} /$ $\left.\mathrm{m}^{2}\right)$ e ciclofosfamida $\left(500 \mathrm{mg} / \mathrm{m}^{2}\right)$; HEC=epirrubicina $\left(100 \mathrm{mg} / \mathrm{m}^{2}\right)$ e ciclofosfamida $\left(830 \mathrm{mg} / \mathrm{m}^{2}\right)$; IC=intervalo de confiança

Num terceiro estudo randomizado, investigadores franceses trataram 621 pacientes pré-menopausa, com linfonodos positivos ${ }^{35}$. Nesse estudo, as pacientes receberam FEC 50 por seis ciclos, FEC 50 por três ciclos ou FEC 75 (fluorouracil, $500 \mathrm{mg} / \mathrm{m}^{2}$, epirrubicina, $75 \mathrm{mg} / \mathrm{m}^{2}$ e ciclofosfamida, $500 \mathrm{mg} / \mathrm{m}^{2}$, todos no dia 1 a cada 3 semanas) por três ciclos. O estudo revelou que o tratamento mais prolongado foi mais eficaz (Tabela 3), e não houve diferenças significativas entre os perfis de toxicidade.

Outro tema em estudo é o papel da densificação da dose, que consiste no emprego da mesma dose total de um regime quimioterápico, mas com menor periodicidade. Esse tratamento com ciclos mais curtos, conhecido como tratamento com dose densa, tem seu término acelerado e, potencialmente, sua eficácia aumentada. Recentemente, demonstrou-se que o tratamento em dose densa com o regime $\mathrm{AC}+\mathrm{T}$ (doxorrubicina, ciclofosfamida e paclitaxel) promove

Tabela 1. Comparação direta entre os regimes FEC 50 e FEC $100^{33}$

\begin{tabular}{l|c|c|c|c}
\multicolumn{1}{c|}{ Parâmetro } & FEC 50 & FEC 100 & $\begin{array}{c}\text { p } \\
\text { ajustado* }\end{array}$ & $\begin{array}{c}\text { Risco relativo ajustado } † \\
\text { (IC 95\%) }\end{array}$ \\
\hline Sobrevida livre de doença aos 5 anos & $54,8 \%$ & $63,3 \%$ & 0,02 & $\begin{array}{c}1,37 \\
(1,05-1,79)\end{array}$ \\
\hline Sobrevida global aos 5 anos & $65,3 \%$ & $77,4 \%$ & 0,005 & $\begin{array}{r}1,55 \\
(1,14-2,10)\end{array}$ \\
\hline Neutropenia grau 3 ou 4 & $11,1 \%$ & $25,2 \%$ & $<0,001$ & - \\
\hline Náuseas ou vômitos grau 3 ou 4 & $23,3 \%$ & $34,7 \%$ & 0,008 & - \\
\hline
\end{tabular}

$\left(^{*}\right)$ Ajuste pelo modelo de Cox; $(\dagger)$ Risco relativo de mortalidade entre FEC 50 e FEC 100, ajustado pelo modelo de Cox; FEC=fluorouracil, epirrubicina e ciclofosfamida; IC=intervalo de confiança. 
Tabela 3. Comparação direta entre os regimes FEC 50 e FEC $75^{35}$

\begin{tabular}{l|c|c|c}
\hline \multicolumn{1}{c|}{ Parâmetro } & $\begin{array}{c}\text { FEC } \\
50 \times 6 \\
\text { semanas }\end{array}$ & $\begin{array}{c}\text { FEC } \\
50 \times 3 \\
\text { semanas }\end{array}$ & $\begin{array}{c}\text { FEC } \\
75 \times 3 \\
\text { semanas }\end{array}$ \\
\hline $\begin{array}{l}\text { Sobrevida livre de } \\
\text { doença aos 10 } \\
\text { anos }\end{array}$ & $53,4 \%$ & $42,5 \%$ & $43,6 \%$ \\
\hline $\begin{array}{l}\text { Sobrevida global } \\
\text { aos 10 anos }\end{array}$ & $64,3 \%$ & $56,6 \%$ & $59,7 \%$ \\
\hline
\end{tabular}

FEC=fluorouracil, epirrubicina e ciclofosfamida

aumento da sobrevida livre de doença e da sobrevida global, em mulheres com comprometimento axilar pelo câncer de mama ${ }^{36}$. Estudos semelhantes também vêm sendo conduzidos com a epirrubicina na Europa. Os resultados preliminares de um estudo alemão demonstraram aumento da sobrevida livre de doença e da sobrevida global em pacientes com quatro ou mais linfonodos comprometidos ${ }^{37}$. Da mesma forma, pesquisadores italianos estão comparando o regime CEF convencional, administrado a cada 3 semanas, com o mesmo regime empregado a cada 2 semanas $^{38}$. Foram publicados apenas resultados de qualidade de vida, que sugerem que o regime convencional causa menos efeitos colaterais e menos estresse psicológico do que o regime mais denso.

\section{DISCUSSÃO}

Os estudos publicados até o presente momento não permitem uma conclusão definitiva sobre a dose mais adequada da epirrubicina no tratamento adjuvante do câncer de mama. As comparações diretas em estudos randomizados demonstraram que o regime FEC 100 é superior ao regime FEC $50^{33}$, e que o tratamento com três ciclos do regime FEC 75 é inferior ao tratamento com seis ciclos do regime FEC $50^{35}$. O aumento da dose da epirrubicina de $60 \mathrm{mg} / \mathrm{m}^{2}$ para $100 \mathrm{mg} / \mathrm{m}^{2}$ também promove melhores resultados, mas nesse caso também houve aumento da dose da ciclofosfamida ${ }^{34}$. Os estudos em doença metastática e no tratamento adjuvante mostram que doses mais elevadas estão associadas à maior toxicidade. Este achado tem importância relativa, com implicações diferentes no contexto do tratamento paliativo ou curativo. Para o tratamento paliativo, um dos principais objetivos é priorizar a qualidade de vida, desde que não haja comprometimento da eficácia terapêutica. Esta útltima deve ser avaliada por parâmetros como a sobrevida global e o tempo até a progressão. Por outro lado, o tratamento curativo permite que se aceite maior grau de toxicidade aguda, desde que haja comprovação de maior eficácia em estudos randomizados de grande porte e que não haja toxicidade tardia limitante.

Parece claro que o regime FEC 50 é inadequado e que o tratamento adjuvante deve ser feito por seis ciclos, quando se faz opção por regimes contendo a epirrubicina, mas sem um taxano (paclitaxel ou docetaxel). $\mathrm{O}$ regime FEC 100 foi adotado como padrão na Europa, onde está sendo conduzido o estudo PACS-1 ${ }^{39}$. Esse estudo compara seis ciclos de FEC 100 a três ciclos de FEC 100 seguidos de três ciclos de docetaxel. Em virtude do benefício conferido pelo docetaxel, quando associado à doxorrubicina ${ }^{40} \mathrm{e}$ do papel atual dos taxanos no tratamento adjuvante de pacientes com linfonodos positivos ${ }^{36,40-42}$ é possível que ainda haja modificações dos regimes de tratamento adjuvante que contêm a epirrubicina.

\section{CONCLUSÕES}

Em nossa opinião, o regime ideal com a epirrubicina ainda não foi definido. Neste serviço, utilizam-se regimes com epirrubicina ou doxorrubicina. Nos casos em que se opta pela epirrubicina, propóe-se a utilização dos regimes FEC 75 ou FEC 100, que são administrados a cada 21 dias. Em pacientes com linfonodos negativos, em tratamento adjuvante, o regime FEC 75 é administrado por seis ciclos; para pacientes com doença localmente avançada, emprega-se o regime FEC 100. Para aquelas com linfonodos comprometidos pelo tumor, procura-se tratá-las também com o paclitaxel, o taxano mais amplamente disponível na rede pública.

\section{REFERÊNCIAS}

1. World Health Organization (WHO). International Agency for Research on Cancer. World Cancer Report. Lyon: IARC Press; 2003:188-93.

2. Ministério da Saúde. Instituto Nacional de Câncer. Estimativa 2005 [homepage na internet]. Incidência de Câncer no Brasil. [acesso em abril 2005]. Disponível em <http://www.inca.gov.br/estimativa/2005>

3. Gonçalves PHB, Gaui MF, Martins RG, Bines J. Padrão de tratamento cirúrgico do câncer de mama de acordo com a idade - Análise de 5 anos do Instituto Nacional de Câncer (INCA). Trabalho apresentado no XVI Congresso Brasileiro de Cancerologia e XIII Congresso Brasileiro de Oncologia Clínica; 2003 novembro 26 a 30; São Paulo, Brasil.

4. Early Breast Cancer Trialists' Collaborative Group. Tamoxifen for early breast cancer: an overview of the randomised trials. Lancet. 1998;351:1451-467. 
5. Early Breast Cancer Trialists' Collaborative Group. Polychemotherapy for early breast cancer: an overview of the randomised trials. Lancet. 1998;352:930-42.

6. Bonfante V, Bonadonna G, Villani F, Martini A. Preliminary clinical experience with 4-epidoxorubicin in advanced human neoplasia. Recent Results Cancer Res. 1980;74:192-99.

7. Hill BT, Whelan RD. A comparison of the lethal and kinetic effects of doxorubicin and 4'-epi-doxorubicin in vitro. Tumori. 1982;68:29-37.

8. Taetle R, Howell SB, Giuliani FC, Koziol J, Koessler A. Comparison of the activity of doxorubicin analogues using colony-forming assays and human xenografts. Cancer. 1982;50:1455-461.

9. Bonfante V, Villani F, Bonadonna G. Toxic and therapeutic activity of 4'-epi-doxorubicin. Tumori.1982;68:105-11.

10. Fossa SD, Wik B, Bae E, Lien HH. Phase II study of 4'-epidoxorubicin in metastatic renal cancer. Cancer Treat Rep. 1982;66:1219-221.

11. Kolaric K, Potrebica V, Cervek J. Phase-II clinical trial of 4'epi-doxorubicin in metastatic solid tumors. J Cancer Res Clin Oncol. 1983;106:148-52.

12. Robustelli Della Cuna G, Pavesi L, Preti P, Ganzina F. Clinical evaluation of 4'-epi-doxorubicin in advanced solid tumors. Invest New Drugs. 1983;1:349-53.

13. Ganzina F. 4'-epi-doxorubicin, a new analogue of doxorubicin: a preliminary overview of preclinical and clinical data. Cancer Treat Rev. 1983;10:1-22.

14. Villani F, Comazzi R, Lacaita G, et al. Preliminary echocardiographic and polygraphic evaluation of cardiac toxicity of 4'-epi-doxorubicin. Int J Clin Pharmacol Ther Toxicol. 1983;21:203-208.

15. Jain KK, Casper ES, Geller NL, Hakes TB, Kaufman RJ, Currie $\mathrm{V}$ et al. A prospective randomized comparison of epirubicin and doxorubicin in patients with advanced breast cancer. J Clin Oncol. 1985;3:818-26.

16. Robert J. Epirubicin: clinical pharmacology and dose-effect relationship. Drugs. 1993;45(suppl 2):20-30.

17. Torti FM, Bristow MM, Lum BL, Carter SK, Howes AE, Aston DA et al. Cardiotoxicity of epirubicin and doxorubicin: assessment by endomyocardial biopsy. Cancer Res. 1986;46:3722-727.

18. Coukell AJ, Faulds D. Epirubicin: An updated review of its pharmacodynamic and pharmacokinetic properties and therapeutic efficacy in the management of breast cancer. Drugs. 1997;53:453-82.

19. Habeshaw T, Paul J, Jones R, Stallard S, Stewart M, Kaye $\mathrm{SB}$ et al. Epirubicin at two dose levels with prednisolone as treatment for advanced breast cancer: the results of a randomized trial. J Clin Oncol. 1991;9:295-304.

20. French Epirubicin Study Group. A prospective randomized trial comparing epirubicin monochemotherapy to two fluorouracil, cyclophosphamide, and epirubicin regimens differing in epirubicin dose in advanced breast cancer patients. J Clin Oncol. 1991;9:305-12.

21. Focan C, Andrien JM, Closon MT, Dicato M, Driesschaert P, Focan-Henrard D et al. Dose-response relationship of epirubicin-based first-line chemotherapy for advanced breast cancer: a prospective randomized trial. J Clin Oncol. 1993;11:1253-263.

22. Brufman G, Colajori E, Ghilezan N, Lassus M, Martoni A, Perevodchikova $\mathrm{N}$ et al. Doubling epirubicin dose intensity $(100 \mathrm{mg} / \mathrm{m} 2$ versus $50 \mathrm{mg} / \mathrm{m} 2)$ in the $\mathrm{FEC}$ regimen significantly increases response rates. An international randomised phase III study in metastatic breast cancer. The Epirubicin High Dose (HEPI 010) Study Group. Ann Oncol. 1997;8:155-62.

23. Fountzilas G, Athanassiades A, Giannakakis T, Briasoulis E, Bafaloukos D, Kalogera-Fountzila A et al. A randomized study of epirubicin monotherapy every four or every two weeks in advanced breast cancer. A Hellenic Cooperative Oncology Group study. Ann Oncol. 1997;8:1213-220.

24. Bastholt L, Dalmark M, Gjedde SB, Pfeiffer P, Pedersen D, Sandberg E et al. Dose-response relationship of epirubicin in the treatment of postmenopausal patients with metastatic breast cancer: a randomized study of epirubicin at four different dose levels performed by the Danish Breast Cancer Cooperative Group. J Clin Oncol. 1996;14:1146-155.

25. Blomqvist C, Elomaa I, Rissanen P, Hietanen P, Nevasaari $\mathrm{K}$, Helle L. Influence of treatment schedule on toxicity and efficacy of cyclophosphamide, epirubicin, and fluorouracil in metastatic breast cancer: a randomized trial comparing weekly and every-4-week administration. J Clin Oncol. 1993;11:467-73.

26. Fossati R, Confalonieri C, Torri V, Ghislandi E, Penna A, Pistotti $\mathrm{V}$ et al. Cytotoxic and hormonal treatment for metastatic breast cancer: a systematic review of published randomized trials involving 31,510 women. J Clin Oncol. 1998; 16:3439-460.

27. Therasse P, Mauriac L, Welnicka-Jaskiewicz M, Bruning P, Cufer T, Bonnefoi $\mathrm{H}$ et al. Final results of a randomized phase III trial comparing cyclophosphamide, epirubicin, and fluorouracil with a dose-intensified epirubicin and cyclophosphamide + filgrastim as neoadjuvant treatment in locally advanced breast cancer: an EORTC-NCIC-SAKK Multicenter Study. J Clin Oncol. 2003;21:843-50.

28. Smith IE, A'Hern RP, Coombes GA, Howell A, Ebbs SR, Hickish TF et al. A novel continuous infusional 5fluorouracil-based chemotherapy regimen compared with conventional chemotherapy in the neo-adjuvant treatment of early breast cancer: 5 year results of the TOPIC trial. Ann Oncol. 2004;15:751-58.

29. Cocconi G, Di Blasio B, Boni C, Bisagni G, Ceci G, Rondini $\mathrm{E}$ et al. Randomized trial comparing cyclophosphamide, methotrexate, and 5-fluorouracil (CMF) with rotational CMF, epirubicin and vincristine as primary chemotherapy in operable breast carcinoma. Cancer. 2002;95:228-35. 
30. Early Breast Cancer Trialists' Collaborative Group. Effects of chemotherapy and hormonal therapy for early breast cancer on recurrence and 15-year survival: an overview of the randomised trials. Lancet. 2005;365:1687-717.

31. Bonadonna G, Brusamolino E, Valagussa P, Rossi A, Brugnatelli L, Brambilla $\mathrm{C}$ et al. Combination chemotherapy as an adjuvant treatment in operable breast cancer. N Engl J Med.1976;294:405-10.

32. Levine MN, Bramwell VH, Pritchard KI, Norris BD, Shepherd LE, Abu-Zahra $\mathrm{H}$ et al. Randomized trial of intensive cyclophosphamide, epirubicin, and fluorouracil chemotherapy compared with cyclophosphamide, methotrexate, and fluorouracil in premenopausal women with node-positive breast cancer. National Cancer Institute of Canada Clinical Trials Group. J Clin Oncol. 1998;16:2651-658.

33. French Adjuvant Study Group. Benefit of a high-dose epirubicin regimen in adjuvant chemotherapy for nodepositive breast cancer patients with poor prognostic factors: 5-year follow-up results of French Adjuvant Study Group 05 randomized trial. J Clin Oncol. 2001;19:602-11.

34. Piccart MJ, Di Leo A, Beauduin M, Vindevoghel A, Michel J, Focan C et al. Phase III trial comparing two dose levels of epirubicin combined with cyclophosphamide with cyclophosphamide, methotrexate, and fluorouracil in nodepositive breast cancer. J Clin Oncol. 2001;19:3103-110.

35. Fumoleau P, Kerbrat P, Romestaing P, Fargeot P, Bremond A, Namer $\mathrm{M}$ et al. Randomized trial comparing six versus three cycles of epirubicin-based adjuvant chemotherapy in premenopausal, node-positive breast cancer patients: 10year follow-up results of the French Adjuvant Study Group 01 trial. J Clin Oncol. 2003;21:298-305.

36. Citron ML, Berry DA, Cirrincione C, Hudis C, Winer EP, Gradishar WJ et al. Randomized trial of dose-dense versus conventionally scheduled and sequential versus concurrent combination chemotherapy as postoperative adjuvant treatment of node-positive primary breast cancer: first report of Intergroup Trial C9741/Cancer and Leukemia Group B Trial 9741. Clin Oncol. 2003;21:1431-439.

37. MöbusVJ, Untch M, du Bois A, Leuck HJ, Thomssen C, Kuhn W, et al. Dose-dense sequential chemotherapy with epirubicin(E), paclitaxel (T) and cyclophosphamide (C) (ETC) is superior to conventional dosed chemotherapy in high-risk breast cancer patients $(=4+\mathrm{LN})$. First results of an AGO-trial. Proc Am Soc Clin Oncol. 2004;22:14S (latebreaking abstract 513).

38. Del Mastro L, Costantini M, Morasso G, Bonci F, Bergaglio $\mathrm{M}$, Banducci $S$ et al. Impact of two different dose-intensity chemotherapy regimens on psychological distress in early breast cancer patients. Eur J Cancer. 2002;38:359-66.

39. Roché H, Fumoleau P, Spielmann M, et al. Five years analysis of the PACS 01 trial: 6 cycles of FEC100 vs 3 cycles of FEC100 followed by 3 cycles of docetaxel (D) for the adjuvant treatment of node positive breast cancer. [Abstract 27]. Proceedings of the San Antonio Breast Cancer Symposium; 2004 Dec 8-11; San Antonio, TX, USA.

40. Martin M, Pienkowski T, Mackey J, et al. Adjuvant docetaxel for node-positive breast cancer. N Engl J Med. 2005;352:2302-313.

41. Mamounas EP, Bryant J, Lembersky BC, et al. Paclitaxel after doxorubicin plus cyclophosphamide as adjuvant chemotherapy for node-positive breast cancer: results from NSABP B-28. J Clin Oncol. 2005;23:3686-696.

42. Henderson IC, Berry DA, Demetri GD, et al. Improved outcomes from adding sequential paclitaxel but not from escalating doxorubicin dose in an adjuvant chemotherapy regimen for patients with node-positive primary breast cancer. J Clin Oncol. 2003;21:976-83.

\section{Abstract}

Epirubicin is a doxorubicin analog that is increasingly used in the management of patients with early and advanced breast cancer. Epirubicin is less cardiotoxic than doxorubicin, thus providing a convenient alternative to the parent compound. Studies in early and advanced breast cancer suggest that these two anthracyclines can be used interchangeably, although the exact dose equivalence between epirubicin and doxorubicin has not been determined. In adjuvant treatment, which aims at cure, studies reported thus far have been unable to establish the most active dose or the best combination containing epirubicin. This article provides an overview of randomized trials and meta-analyses that have attempted to define the role of epirubicin in curative and palliative chemotherapy for patients with breast cancer. Our main question concerns the ideal dose of epirubicin in adjuvant treatment, and we conclude by suggesting the chemotherapy regimen containing epirubicin that we consider most suitable.

Key words: Antibiotics; Antineoplastic; Anthracyclines; Breast cancer; Doxorubicin; Chemotherapy; Epirubicin. 